\title{
Changes in treatment status of patients with severe mental illness in rural China, 1994-2015
}

Mao-Sheng Ran, Xue Weng, Yu-Jun Liu, Tian-Ming Zhang, Yue-Hui Yu, Man-Man Peng, Wei Luo, Shi-Hui Hu, Xin Yang, Bo Liu, Tin Zhang, Graham Thornicroft, Cecilia Lai-Wan Chan and Meng-Ze Xiang

\section{Background}

Although it is crucial to improve the treatment status of people with severe mental illness (SMI), it is still unknown whether and how socioeconomic development influences their treatment status.

\section{Aims}

To explore the change in treatment status in people with SM from 1994 to 2015 in rural China and to examine the factors influencing treatment status in those with SMI.

\section{Method}

Two mental health surveys using identical methods and ICD-10 were conducted in 1994 and 2015 (population $\geq 15$ years old, $n=$ 152 776) in the same six townships of Xinjin County, Chengdu, China.

\section{Results}

Compared with 1994, individuals with SMI in 2015 had significantly higher rates of poor family economic status, fewer family caregivers, longer duration of illness, later age at first onset and poor mental status. Participants in 2015 had significantly higher rates of never being treated, taking antipsychotic drugs and ever being admitted to hospital, and lower rates of using traditional Chinese medicine or being treated by traditional/spiritual healers. The factors strongly associated with never being treated included worse mental status (symptoms/social functioning), older age, having no family caregivers and poor family economic status.

\section{Conclusions}

Socioeconomic development influences the treatment status of people with SMI in contemporary rural China. Relative poverty, having no family caregivers and older age are important factors associated with a worse treatment status. Culture-specific, community-based interventions and targeted poverty-alleviation programmes should be developed to improve the early identification, treatment and recovery of individuals with SMI in rural China.

\section{Declaration of interest}

None.

\section{Keywords}

Socioeconomic status; treatment status; severe mental illness; family caregivers; China.

\section{Copyright and usage}

(c) The Royal College of Psychiatrists 2019. This is an Open Access article, distributed under the terms of the Creative Commons Attribution-NonCommercial-NoDerivatives licence (http://creativecommons.org/licenses/by-nc-nd/4.0/), which permits noncommercial re-use, distribution, and reproduction in any medium, provided the original work is unaltered and is properly cited. The written permission of Cambridge University Press must be obtained for commercial re-use or in order to create a derivative work.
China has experienced profound socioeconomic transformations in recent decades. ${ }^{1}$ The rapid socioeconomic development associated with increased urbanisation and migration may have a negative impact on family systems, which can have significant adverse consequences for individuals with severe mental illness (SMI). ${ }^{2,3} \mathrm{SMI}$, a leading cause of disability, is characterised in this study as mental, behavioural or emotional disorders (including schizophrenia, bipolar disorder and major depressive disorder) that can result in serious functional impairment. ${ }^{4}$ In the Chinese population, the burden of SMI is profoundly high during a period of rapid social development. ${ }^{5}$

\section{Importance of treatment for people with SMI}

How to reduce the large gap between the need for treatment for mental disorders and the provision of that treatment, especially in China, the most populous country in the world, is an important public health issue. ${ }^{3,6,7}$ Receiving appropriate and timely treatment is critical for reducing the burden of mental disorders. Evidence shows that structured treatment and antipsychotic medications can significantly reduce illness symptoms, chronic disability, suicidal rates and healthcare costs. ${ }^{8}$ Absence and delay in treatment for individuals with SMI can lead to severe consequences, including excess mortality, poor prognosis, as well as ongoing functional impairment and work loss. ${ }^{7,8}$ Thus, understanding treatment status and its predictors is crucial for enhancing mental health system and services, and improving outcomes for those with SMI. ${ }^{6,9}$ However little is known about the impact of social development on treatment status for those with SMI in the world. ${ }^{10}$

Following rapid socioeconomic development in China, shifts in the traditional family structure, the acceleration of rural-to-urban migration, and the scarcity of mental health services have resulted in higher prevalence of untreated mental illness in China. ${ }^{3,7}$ Given that less than $1 \%$ of the national healthcare budget is allocated to mental health and the majority of mental health services and resources are concentrated in urban cities, developing ways to increase support networks and strengthen healthcare provision for people with SMI, especially in rural China, has become an urgent public health issue. ${ }^{6,11,12}$ Identifying and overcoming barriers to treatment are essential for improving unmet treatment needs and reducing the substantial burden of mental disorders. ${ }^{13}$

\section{Framework of social development and treatment status of mental illness}

Although Andersen's behavioural model has been the theoretical framework most widely used for studying the utilisation of health services, ${ }^{14}$ little is known about whether and how long-term social development factors (for example socioeconomic, urbanisation, migration) influence treatment status through predisposing characteristics (such as gender, age, marital status, family size), enabling factors (such as family income, type of care) and need indicators (such as symptoms, diagnoses) in people with SMI. ${ }^{15}$ 


\section{Changes in treatment status of people with SMI in rural China}

The majority of previous studies on treatment status in people with SMI have been from high-income or Western contexts. ${ }^{9}$ Few studies had been conducted to explore the treatment status of people with SMI in China, especially rural China, where limited healthcare resources are available., ${ }^{2,3}$ The relationship between poverty and mental health is a central concern of global poverty and is of great interest to both health and economic policymakers. ${ }^{16-18}$ In China, poverty may be an important factor influencing treatment status. ${ }^{19,20}$ Most individuals with mental disorders (over $90 \%$ ) live with their family members who are their primary caregivers. ${ }^{6,21}$ However, it is still unknown whether changes in poverty status and in family caregivers during a period of rapid socioeconomic development in China has had an impact on the treatment status of people with SMI.

Since the 1980s China has experienced dramatic socioeconomic change, which is reflected in the steady rise in gross domestic product (GDP). The fastest growth period for GDP in China was from 1994 to 2015, during this time the growth rate in GDP was remarkable, reaching $1316.7 \%{ }^{1}$ Similar to many other counties in China, Xinjin County, in the south of Chengdu, has also undergone rapid socioeconomic development and urbanisation since the 1980s. The average net income of each person in Xinjin had increased from 1330 RMB in 1994 to 16856 RMB in 2015 (percentage increase: $1167.4 \%){ }^{22}$ Although China's rapid economic growth (such as GDP) in the past three decades has produced many economic benefits, raising the average family income and pulling hundreds of millions of people out of poverty, it is still unknown whether or how the treatment status of individuals with SMI has been influenced by this socioeconomic development. Chengdu Mental Health Project (CMHP), an ongoing longitudinal mental health project, provides a unique opportunity to explore the impact of social development on the treatment status of individuals with SMI in rural China. ${ }^{2}$

\section{Study objectives}

This is the first study to explore the relationship between social development (socioeconomic, urbanisation and migration) and treatment status using the data from two mental health surveys (1994 and 2015) in people with SMI in rural China. The hypotheses tested in this study are: (a) social development will influence the treatment status of people with SMI; and (b) changes in family circumstances (such as family size, income and family caregivers) will have an impact upon the treatment status of individuals with SMI in the community. The objectives of this study were to: (a) explore the changes in treatment status of individuals with SMI from 1994 to 2015 in rural China; and (b) examine factors influencing treatment status.

\section{Method}

\section{Study population and procedures}

The study data are derived from the CMHP, a longitudinal study on mental illness and mental health services in Xinjin County, Chengdu, which is a representative middle-income rural county in Southwest China. ${ }^{2,7,21}$ People with SMI (with schizophrenia, or mood disorders (bipolar disorder or major depressive disorder)) were identified from two mental health surveys on mental disorders and mental health services in the population who were over 15 years old in the same six townships of Xinjin County, Chengdu, China in 1994 (population: 123 572) and 2015 (population: 152 776) respectively (Fig. 1). The six townships were randomly selected from all 12 townships of Xinjin County in 1994 (total population frame: 279700$)^{\text {. }}$

The methods of the two mental health surveys in 1994 and 2015 were similar and have been described in detail elsewhere. ${ }^{2,7,20,21}$ Briefly, all individuals with mental disorders were identified through two steps: (a) screening procedures for psychosis (faceto-face interviews with the head of each household together with the key informant method) one household by household (as such, the sample screened equalled the response rate of $98.0 \% \times$ all households in the six random townships), and (b) general psychiatric interview. First, face-to-face interviews were conducted by trained investigators (for example nurses, psychologists or social workers) with each head of household (together with the key-informant method) to identify potential individuals with cases of mental disorder. Another household resident (priority list: parent, spouse,

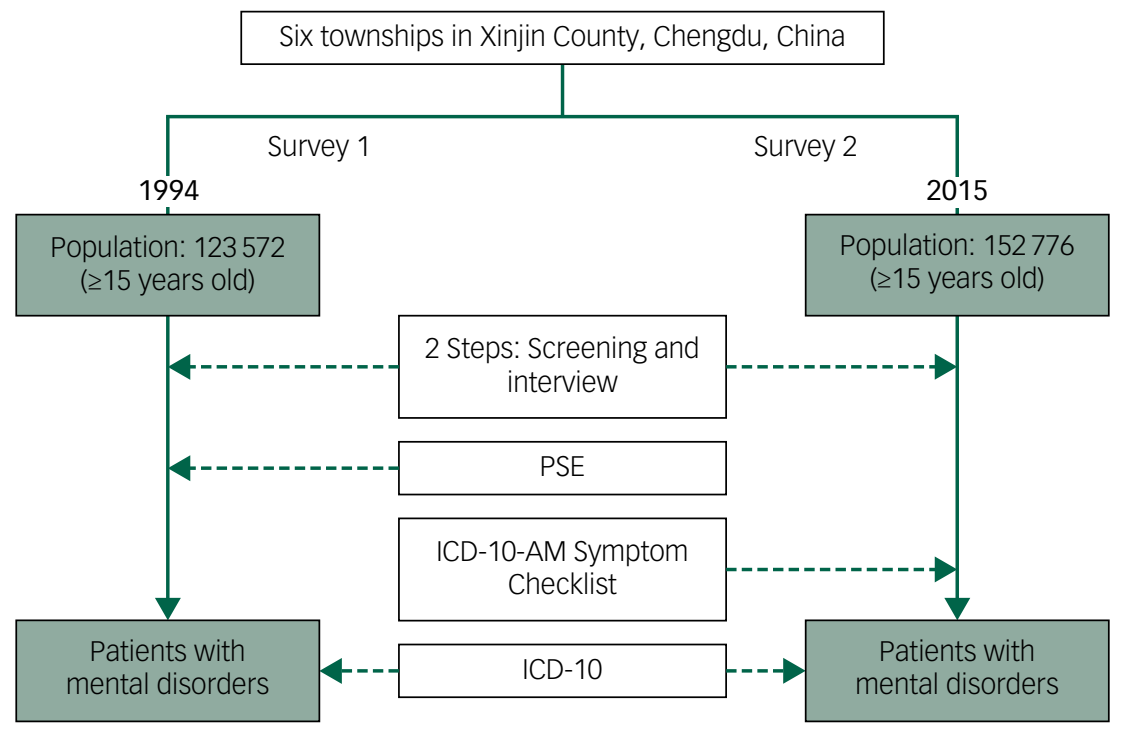

Fig. 1 Study design and method. 


$\begin{array}{cr}2015 & \text { Differences, } \\ 68905.2 & \uparrow 1316.7 \\ 23018.0 & \uparrow 2189.0 \\ 16856.0^{* * *} & \uparrow 1167.4 \\ 8420.1^{* * *} & \uparrow 658.6 \\ 56.1 & \uparrow 96.8 \\ 53.8 & \uparrow 167.7\end{array}$

offspring or others) was selected for the interview if the head of household could not be interviewed. The Psychoses Screening Schedule (PSS) ${ }^{23}$ was completed by means of interviewing the heads of all the households and discussion with village doctors and neighbourhoods comprising the survey sample. Second, once an individual was identified as a potential patient with psychosis, trained psychiatrists conducted a comprehensive general psychiatric interview with that person for further diagnosis. The inclusive and exclusive criteria of the survey population were the same in the two surveys in 1994 and 2015. The two mental health surveys ${ }^{2,7}$ used the same diagnostic criteria, the ICD-10. ${ }^{24}$ The field procedures and quality controls were strictly maintained by the surveys team under supervision from the CMHP. Diagnostic reliability had been established in the trained research interviewers including attending psychiatrists with over 5 years clinical experience. The two surveys were approved by the University Human Research Ethics Committee (HREC; 1994: West China University of Medical Sciences, 2015: The University of Hong Kong) and all participants gave informed consent at each stage of the study.

\section{Measurement}

The standardised assessment tools included the PSS, Present State Examination (PSE-9), ${ }^{25}$ Social Disability Screening Schedule $(\mathrm{SDSS})^{26}$ and the General Psychiatric Interview Schedule ${ }^{27}$ (including treatment status) in the mental health survey in $1994 .^{7,20}$ The standardised assessment tools mainly included the PSS, ICD-10AM Symptom Checklist for Mental Disorders (ICD-10-ChecklistAM) ${ }^{28}$ Positive and Negative Syndrome Scale, ${ }^{29}$ SDSS, Global Assessment of Functioning ${ }^{30}$ and the General Psychiatric Interview Schedule (including treatment status) in the mental health survey in 2015. ${ }^{2,31}$ After the training session, all the assessment tools reached an acceptable level of reliability between research interviewers (kappa $(\kappa)>0.74$ in 1994 and $\kappa>0.75$ in 2015). All interviews were conducted by trained psychiatrists using the standardised assessment tools. In the event of a disagreement, a team of research psychiatrists with over 10 years' clinical experience reviewed the participant's case to establish the final diagnosis.

\section{Statistical analysis}

Cross-tabulation was performed to compare the economic status and urbanisation rate in China and Xinjin County in 1994 and 2015. Participants' demographic, socioeconomic, psychological outcomes in 1994 and 2015 were assessed using chi-square $\left(\chi^{2}\right)$ tests for categorical variables and paired samples $t$-tests for continuous variables normally distributed. Participants' treatment status in 1994 and 2015 were assessed using $\chi^{2}$ tests. A logistic regression model (stepwise) was used to analyse the factors associated with nevertreated status in 1994 and 2015. The never-treated status (never being treated or only once treated with medication) was considered as the dependent variable, whereas the other variables (such as gender, age, family caregivers, family history of mental illness, family economic status, duration of illness, present mental status, etc.) were the independent variables. Both backward and forward inclusions of variables in the logistic regression equation were tested to identify the most stable model. All statistical tests were two-tailed with a significance level of 0.05 . All statistical analyses were conducted using SPSS statistical software (IBM SPSS Statistics for Windows, Version 24.0).

\section{Results}

\section{Socioeconomic status}

Table 1 shows the socioeconomic status and urbanisation rate from 1994 to 2015. GDP in China and Xinjin, Chengdu from 1994 to 2015 has increased $1416.7 \%$ and $2289.0 \%$, respectively. The urbanisation rate in Xinjin increased from $20.1 \%$ in 1994 to $53.8 \%$ in 2015, which is similar to the China average. Compared with the annual net income per person in the population in Xinjin, Chengdu, the annual net income per person in participants with SMI was significantly lower in both 1994 and $2015(P<0.001)$, and the rate of different annual net income per person between the population and participants with SMI was $19.8 \%$ in 1994 and $100.2 \%$ in 2015, respectively (Fig. 2). The rate of poor family economic status (less than mean level) was significantly higher in 2015 (65.2\%) than that in $1994(48.2 \%)(P<0.001)$ (Table 2).

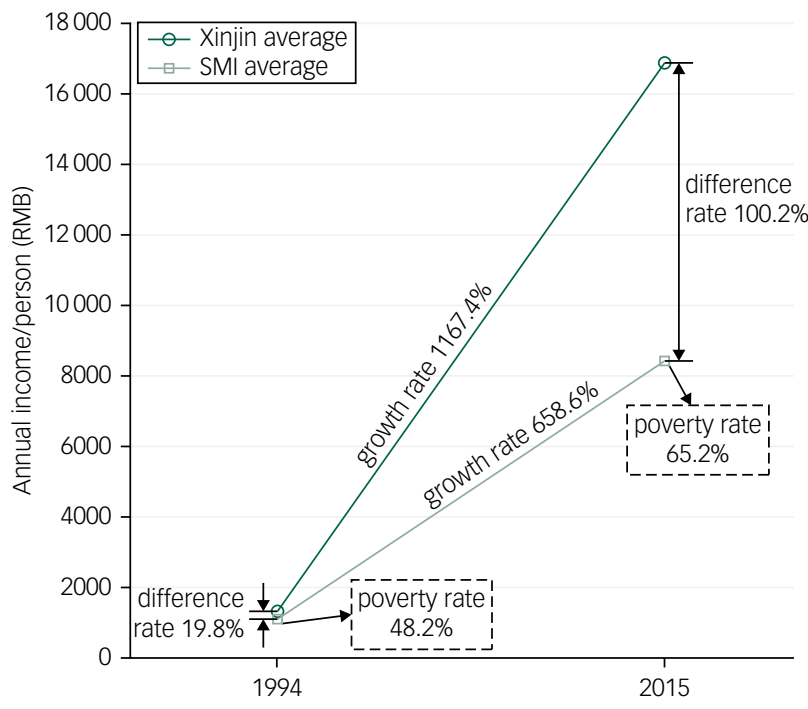

Fig. 2 Difference in annual income per person in 1994 and 2015 in the population in Xinjin compared with people with severe mental illness. 


\section{Characteristics of participants with SMI in 1994 and 2015}

Table 2 shows the characteristics of participants with SMI in 1994 and 2015. In 1994, there were 711 people with SMI (schizophrenia: 515 (72.4\%), and mood disorders: 196 (27.6\%)). In 2015, there were 1042 participants with SMI (schizophrenia: 671 (64.4\%), and mood disorders: $371(35.6 \%))\left(\chi^{2}=12.5\right.$, d.f. $\left.=1, P<0.001\right)$. Compared with participants with SMI in 1994, participants with SMI in 2015 were significantly older, and had significantly fewer family members, an older age at first onset, a longer duration of illness, a lower rate of family history of mental illness, a higher rate of being without family caregivers, a higher rate of poor family economic status (compared with the average family in the village) and lower rates of full and partial remission $(P<0.001)$.

\section{Treatment status in participants with SMI in 1994 and 2015}

Table 3 shows the treatment status for participants with SMI in 1994 and 2015. Compared with participants with SMI in 1994, participants in 2015 had significantly higher rates of never being treated, taking antipsychotic drugs and ever being admitted to hospital and lower rates of taking antipsychotic drugs for $\leq 2$ months, ever being treated with traditional Chinese medicine, and ever being treated by traditional/spiritual healers $(P<0.001)$. Compared with participants aged 18-64 years, elderly participants (age $\geq 65$ years) had significantly higher rates of never being treated, and lower rates of ever being admitted to hospital in 1994 and $2015(P<0.001)$. Elderly participants (age $\geq 65$ years) had significantly lower rates of taking antipsychotic drugs for more than 1 year and taking antipsychotic drugs than those aged $18-64$ years in $2015(P<0.001)$.

\section{Factors associated with the never-treated status in participants with SMI}

Table 4 shows the factors associated with the never-treated status for participants with SMI in 1994 and 2015. The factors associated with never-treated status in the final logistic regression model included poor mental status (marked symptoms/deteriorated), older age, no family caregivers and shorter duration of illness (years) in 1994 and $2015(P<0.05$ or $<0.001)$. Moreover, the factors associated with never-treated status in the final logistic regression model also included no family history of mental illness and poor family economic status (less than mean level) in $2015(P<0.05)$.

\section{Discussion}

To the best of our knowledge, this is the first study to compare the 21-year (1994-2015) change in treatment status for people with SMI in a Chinese rural community. ${ }^{2}$ This study contributes rigorous empirical evidence on the change in, and factors associated with, treatment status in individuals with SMI in rural China. The strengths of this study include the use of two mental health surveys with large representative community samples, identical methods and diagnostic criteria in the same rural area in China, and its 21-year (1994-2015) comparative data.

\section{Change in treatment status from 1994 to 2015}

The results of this study indicated that participants in 2015 had significantly higher rates of never being treated, taking antipsychotic drugs and ever being admitted to hospital, and had significantly lower rates of taking antipsychotic drugs for $\leq 2$ months than those in 1994. The results indicate that treatment with antipsychotic drugs has improved significantly, but it is still a crucial issue to reduce further the number of people with SMI who have never received treatment (28.3\% in 1994 and $37.2 \%$ in 2015) in rural China. ${ }^{7}$ The rate of never being treated with antipsychotic drugs of $28.3 \%$ in 1994 is consistent with a study in four provinces in China during 2001-2005 (27.6\%). ${ }^{3}$

The results in our study show that the rate of ever being admitted to hospital in individuals with SMI increased from $24.2 \%$ in 1994 to $36.5 \%$ in 2015 . Compared with Western countries, the rates of ever being admitted to hospital and taking antipsychotic drugs in people with SMI are still very low in rural China. ${ }^{32,33}$ Possible reasons may be related to the patients' poor family economic level, lack of knowledge of mental illness and health disparity between rural and urban areas in China (for example most available health resources and services are concentrated in urban-based psychiatric hospitals) $.3,7,21$

Table 2 Characteristics of people with severe mental illness in 1994 and 2015

\begin{tabular}{|c|c|c|}
\hline & $\begin{array}{l}1994 \\
(n=711)\end{array}$ & $\begin{array}{l}2015 \\
(n=1042)\end{array}$ \\
\hline Gender, men: $n(\%)$ & $338(47.5)$ & $471(45.2)$ \\
\hline Married, $n(\%)$ & $471(66.2)$ & $651(62.5)$ \\
\hline Family economic status (less than mean level), $n$ (\%) & $343(48.2)$ & $679(65.2)^{\star * *}$ \\
\hline With family history of mental illness, $n$ (\%) & $202(28.4)$ & $152(14.6)^{\star \star *}$ \\
\hline With family member(s) who worked in other areas over half a year, ${ }^{b} n(\%)$ & - & $198(19.0)$ \\
\hline \multicolumn{3}{|l|}{ Family caregivers, $n(\%)$} \\
\hline Parents & $137(19.3)$ & $145(13.9)^{\star * *}$ \\
\hline Spouse & $378(53.2)$ & $573(55.0)$ \\
\hline Others & $127(17.9)$ & $156(15.0)$ \\
\hline Without caregivers & $69(9.7)$ & $168(16.1)$ \\
\hline \multicolumn{3}{|l|}{ Present mental status, ${ }^{a} n(\%)$} \\
\hline Full remission & $178(25.0)$ & $161(15.5)^{\star \star *}$ \\
\hline Partial remission & $170(23.9)$ & $225(21.6)$ \\
\hline Marked symptoms/deteriorated & $363(51.1)$ & $656(63.0)$ \\
\hline Age, years: mean (s.d.) & $44.5(15.6)$ & $56.0(14.2)^{\star \star *}$ \\
\hline Educational level, years: mean (s.d.) & $5.2(1.2)$ & $5.3(3.6)$ \\
\hline Number of family members, mean (s.d.) & $3.4(1.5)$ & $3.0(1.5)^{\star \star \star}$ \\
\hline Annual net income per person (RMB), mean (s.d.) & $1110(923.3)$ & $8420.1(7927.1)^{* * *}$ \\
\hline Age at first onset, years: mean (s.d.) & $30.6(12.9)$ & $38.0(16.1)^{\star \star \star}$ \\
\hline Duration of illness, years: mean (s.d.) & $12.3(11.7)$ & $17.3(13.8)^{\star \star *}$ \\
\hline
\end{tabular}


Table 3 Treatment status in people with severe mental illness in 1994 and 2015

\begin{tabular}{|c|c|c|c|c|c|c|}
\hline & \multicolumn{3}{|c|}{$1994, n(\%)$} & \multicolumn{3}{|c|}{$2015, n(\%)$} \\
\hline & $\begin{array}{l}\text { Age 18-64 } \\
(n=633)\end{array}$ & $\begin{array}{l}\text { Age } \geq 65 \\
(n=78)\end{array}$ & $\begin{array}{c}\text { Total } \\
(n=711)\end{array}$ & $\begin{array}{c}\text { Age 18-64 } \\
(n=724))\end{array}$ & $\begin{array}{l}\text { Age } \geq 65 \\
(n=318)\end{array}$ & $\begin{array}{c}\text { Total } \\
(n=1042)\end{array}$ \\
\hline Never being treated & $167(26.4)$ & $34(43.6)^{* * *}$ & $201(28.3)$ & $241(33.3)$ & $147(46.2)^{\star \star \star}$ & $388(37.2)^{t+1}$ \\
\hline Antipsychotic drugs $\leq 2$ months & $128(20.2)$ & $14(17.9)$ & $142(20.0)$ & $50(6.9)$ & $31(9.7)$ & $81(7.8)^{t+t}$ \\
\hline Antipsychotic drugs $>1$ year $^{a}$ & - & - & - & $225(31.1)$ & $66(20.8)^{\star * *}$ & $291(27.9)$ \\
\hline Taking antipsychotic drugs & $38(6.0)$ & $1(1.3)$ & $39(5.5)$ & $159(22.0)$ & $39(12.3)^{\star \star \star}$ & $198(19.0)^{\dagger+\dagger}$ \\
\hline Being in mental hospital & $9(1.4)$ & $0(0.0)$ & $9(1.3)$ & $18(2.5)$ & $6(1.9)$ & $24(2.3)$ \\
\hline Ever admitted to hospital & 155 (24.5) & $8(10.3)^{* * *}$ & $163(22.9)$ & $273(37.7)$ & $83(26.1)^{* * *}$ & $356(34.2)^{t+1}$ \\
\hline Ever treatment with traditional Chinese medicine & $430(67.9)$ & $42(53.9)^{\star}$ & $472(66.4)$ & $32(4.4)$ & $22(6.9)$ & $54(5.2)^{\dagger+t}$ \\
\hline Ever treated by traditional/spiritual healers & $331(52.3)$ & $37(47.4)$ & $368(51.8)$ & $98(13.5)$ & $27(8.5)^{*}$ & $125(12.0)^{t+t}$ \\
\hline
\end{tabular}

The results of this study indicated that participants in 2015 had significantly lower rates of ever having been treated with traditional Chinese medicine and by traditional/spiritual healers than that in 1994. Traditionally, the use of traditional/spiritual healers has been common as they are affordable and accessible, especially in Chinese rural areas. ${ }^{34}$ Although little evidence exists to suggest that traditional healers change the course of SMI, individuals with SMI may commonly seek help from traditional/spiritual healers as a means to cope with their illness, which may delay taking medication. ${ }^{35,36}$ The decrease of the rates of ever having been treated with traditional Chinese medicine or by traditional/spiritual healers from 1994 to 2015 may be related to the increase in knowledge about mental illness, the availability of mental health facilities and the change in help-seeking behaviour with socioeconomic development. ${ }^{7,21}$

\section{Economic status and treatment status}

The results of this study showed that the annual net income per person in people with SMI was significantly increased from 1994 to 2015 (658.6\%), but the increase rate was significantly lower than that in all the population of Xinjin, Chengdu (1167.4\%). The annual net income per person with SMI did increase from 1994 to 2015, but the population of Xinjin had a significantly higher increase rate of annual net income, and the rate of poor family economic status (less than the mean level) in those with SMI was significantly higher in 2015 than that in 1994. The findings of this study are consistent with previous studies that indicate the association between SMI and poverty, especially in low- and middle-income countries. ${ }^{37}$ The results of this study also indicate that the family economic status of individuals with SMI has deteriorated and the relative poverty (the level of income in relation to the mean or median income of a population) has become more severe from 1994 to 2015. We suggest that the relative poverty in people with SMI will become more severe with ongoing socioeconomic development (see Ran et al). ${ }^{38}$ Moreover, poor family economic status associated with stigma and changes in life circumstances (for example education, employment) may have a greater impact on mental healthcare, such as resulting in neverbeing treated status, in those with SMI in communities. ${ }^{18,19,38}$
Thus, it is crucial to protect the socioeconomic and environmental status of patients with SMI from declining further and further. ${ }^{37,38}$

\section{Family structure, family caregivers and treatment status}

Traditionally family caregivers are crucial in the care and treatment of individuals with mental disorders in China. ${ }^{21,34}$ The results of this study showed that the rate of those without family caregivers was significantly higher in participants in 2015 (16.1\%) compared with 1994 (9.7\%), and the mean number of family members was significantly decreased from 3.4 (s.d. = 1.5) in 1994 to $3.0($ s.d. $=1.5)$ in 2015. The results indicate the impact of social development on family structure (for example family size) and family caregivers, which may have an impact on healthcare systems in China. Smaller family size and lack of family caregivers were both found to be important factors associated with individuals never being treated, which is consistent with previous studies. ${ }^{21}$ Poor knowledge of mental disorders and stigma associated with mental illness may also prohibit family caregivers and individuals with SMI from seeking treatment. ${ }^{6,18,31}$

\section{Clinical factors and treatment status}

The results of this study showed that participants in 2015 were significantly older at age of first onset, had significantly lower rates of full and partial remission, and longer duration of illness than those in 1994. Moreover, factors associated with never-being treated status in the regression model included poor mental status (symptoms/deteriorated), shorter duration of illness and no family history of mental illness. This indicates that the more severe the symptoms the less likely people with SMI will go to see doctors, but the longer the duration of illness the more chance those with SMI will go to see doctors. This is consistent with a few other studies in China, ${ }^{7,20}$ but inconsistent with studies in Western countries in which the association between greater severity and receipt of treatment among adults was positive and did not differ over time. $^{39,40}$ This may be a specific situation in rural China, and the reasons may include (a) lower awareness and knowledge of and stigma attached to mental illness may prevent individuals from

Table 4 Factors independently associated with the never-treated status for participants with severe mental illness ${ }^{a}$

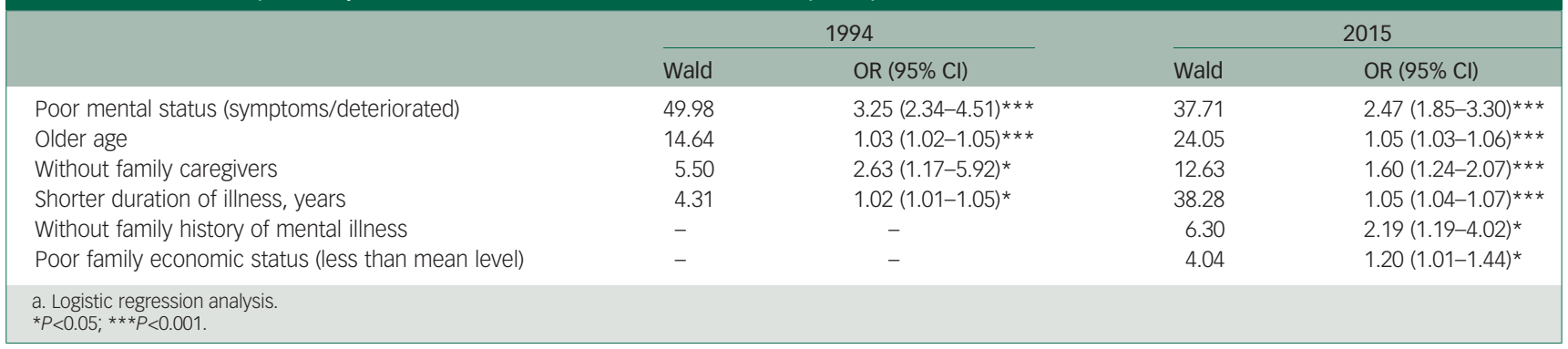


seeking treatment; (b) growing numbers of older people with SMI who may not seek treatment; and (c) poor family economic status, without family caregivers and lack of national health insurance to guarantee treatment for those with SMI in China. ${ }^{7,21,38}$

\section{Other factors and treatment status}

Evidence indicates that levels of treatment for older adults with SMI are very low in general, and younger and older adults with SMI may have different patterns of treatment status. ${ }^{41,42}$ The results of this study also showed that elderly people ( $\geq 65$ years) with SMI had significantly higher rates of never being treated and lower rates of ever being admitted to hospital than younger participants (age 18-64 years) in 1994 and 2015. Older age was maintained in the final logistic regression model for never-treated status, which is consistent with previous studies indicating family members of older people with mental disorders have low mental literacy and are more likely to believe that it is unnecessary to receive treatment. ${ }^{21,42,43}$ Evidence also shows that older people with mental illness face more negative attitudes and are the least likely to report any perceived need for mental healthcare. ${ }^{44,45}$

\section{Social development theory for people with mental illness}

Based on this empirical evidence, we propose a social development theory for people with mental illness. First, with social development, mental health issues including treatment and recovery will gradually become more important. Second, factors related to social development (such as socioeconomic status, urbanisation, migration) may influence the onset of mental illness, family structure (for example family size, knowledge) and family economic status (for example, income, especially level of relative poverty), health status (symptoms and diagnosis), treatment status of individuals with mental illness and the outcome of the mental illness. Social development will influence treatment status (type, length and adherence with treatment) through predisposing, need and enabling factors. ${ }^{14}$ Further studies need to be conducted to test the model.

\section{Limitations of the study}

The limitations of this study include the differences in symptom tools (for example PSE and ICD-10-Checklist-AM) in 1994 and 2015, but the same diagnostic criteria (ICD-10) may minimise the issue. Different attitudes relating to the stigma relating to mental disorders and response bias may affect the responses in the two surveys. Treatment status may be different in people with schizophrenia, bipolar disorder and major depressive disorder and this needs further study. Given the diversity of social and cultural variances in socioeconomic and healthcare characteristics, the results of this study may not be generalisable or replicable in urban areas, other areas with different characteristics in China and other high-income countries.

\section{Implications for policy and services}

The results of this study indicate that social development (for example socioeconomic, urbanisation, migration) may influence the treatment status of individuals with SMI positively or negatively. Understanding and monitoring the change in treatment status and its predictors in people with SMI should be crucial for formulating health policies, enhancing awareness of the importance of treatment, scaling up effective and cost-effective treatments and interventions, and improving mental health recovery. ${ }^{10,16}$ Except hospital-based services, community-based mental health services should be strengthened for people with mental illness in communities. ${ }^{6,10,21}$ Health resources should be equitably distributed in rural and urban areas so that health disparities may be reduced. General physicians and primary health workers (such as village doctors) should be trained to provide community-based services in rural China. ${ }^{3,6,7}$ Developing health policies, national health insurance and interventions to promote access to mental health services (medication and other effective psychosocial interventions), especially in rural China, is crucial for realising the aims of Healthy China 2030. ${ }^{3,31,34}$ Moreover, the long-term goal should be to improve the mental healthcare, treatment and recovery of people with mental illness.

Understanding the impact of social development on mental illness and relevant treatment will enhance effective promotion of mental health. The findings of this study suggest that social development (socioeconomic, urbanisation, migration) may result in the outcome of SMI being more severe (for example lower rates of full and partial remission). Poor mental status (symptoms/deteriorated) may in fact be a barrier to treatment, which may be because of stigmatisation associated with mental illness, especially in the Chinese context. ${ }^{31,46}$ It is an important long-term process to improve access, comprehensiveness and quality of services for those with SMI during a period of social development. Given limited resources, it is crucial to develop early diagnosis, treatment, community-based mental health services and recovery programmes for people with SMI in rural China. ${ }^{6}$

Evidence indicates that mental illness and poverty interact in a negative cycle within low- and middle-income countries. ${ }^{37,38}$ The results of this study indicate that even though socioeconomic development in China may raise the mean family income, it may also result in making relative poverty more severe in people with SMI in the community. Family poverty, especially relative poverty, is significantly associated with never-being treated status. The results of this study provide solid theoretical support for developing targeted poverty-alleviation policies for reducing poverty in people with mental disorders and their families in the community. ${ }^{38} \mathrm{~A}$ national health insurance system and other relevant support programmes (such as occupational training and employment) should be developed to guarantee mental health treatment and recovery for those with SMI. ${ }^{47}$

Evidence indicates that the absence of a family caregiver is a predictive factor for poor long-term outcome for individuals with schizophrenia in a rural community. ${ }^{21}$ The results of this study show that family caregivers play an important role in the care and treatment of those with SMI in rural China. Given the limited social networks for people with SMI, it is crucial to emphasise the role of family caregivers and empower family members in mental health policies, community-based mental health services and family interventions. ${ }^{21,34,48}$

Mao-Sheng Ran, MMed, PhD, Associate Professor, Department of Social Work and Social Administration, University of Hong Kong, China; Xue Weng, PhD, Researcher, Department of Social Work and Social Administration, University of Hong Kong, China; Yu-Jun Liu, PhD, Researcher, Department of Social Work and Social Administration, University of Hong Kong, China; Tian-Ming Zhang, MSW, Researcher, Department of Social Work and Social Administration, University of Hong Kong, China; Yue-Hui Yu, LLM, Researcher, Department of Social Work and Social Administration, University of Hong Kong, China; Man-Man Peng, MASP, Researcher, Department of Social Work and Social Administration, University of Hong Kong, China; Wei Luo, MD, Doctor, Xinjin Mental Hospital, China; Shi-Hui Hu, MD, Doctor, Chengdu Mental Health Center, China; Xin Yang, MD, Doctor, Guangyuan Mental Health Center, China; Bo Liu, MD, Professor, Jingzhou Mental Health Center, China; Tin Zhang, MD, Doctor, Santai Mental Health Center, China; Graham Thornicroft, PhD, Professor, Centre for Global Mental Health Institute of Psychiatry, Psychology and Neuroscience, King's College London, UK; Cecilia Lai-Wan Chan, PhD, Professor, Department of Social Work and Social Administration, University of Hong Kong, China; Meng-Ze Xiang, MD, Professor, Department of Psychiatry, West China Hospital, Sichuan University, China

Correspondence: Mao-Sheng Ran, Department of Social Work and Social Administration, University of Hong Kong, Pokfulam Road, Hong Kong, China. Email: msran@hku.hk

First received 21 Nov 2018, final revision 15 Feb 2019, accepted 18 Feb 2019

\section{Funding}

The mental health survey in 1994 was supported in part by a grant from the China Medical Board in New York (CMB, 92-557; Principal investigator (PI): M.-Z.X.). The mental health survey in 2015 was supported in part by Seed Funding Programme for Basic Research (HKU, 2014-2016, PI: 
M.-S.R), Seed Funding Programme for Applied Research (HKU, 2014-2016, PI: M.-S.R), Strategic Research Theme: Contemporary China Seed Funding (HKU, 2014-2016, PI: M.-S.R), and Menta Health Research in Chengdu, China (Dept. Matching Fund, 2015-2017, PI: M.-S.R).

\section{Acknowledgements}

The authors thank all the collaborative institutes (Xinjin Mental Hospital, Chengdu Mental Health Center, Sichuan Veterans Hospital, Jingzhou Mental Health Center, Guangyuan Mental Health Center, Santai Mental Health Center, Sichuan College of Traditional Chinese Medicine, Southwest Medical University, Chongqing Medical University, Chengdu Mental Hospital, Panzhihua Mental Health Center) and the CMHP Study group for collaboration, support and data collection.

\section{References}

1 World Bank. GDP per Capita. World Bank 2015 (data.woldbank.org/indicator/ NY.GDP.PCAP.CD)

2 Ran MS, Weng X, Liu YJ, Zhang T-M, Thornicroft G, Davidson L, et al. Severe mental disorders in rural China: a longitudinal survey (abstract). Lancet 2017; 390: S37.

3 Phillips MR, Zhang J, Shi Q, Song Z, Ding Z, Pang S, et al. Prevalence, treatment and associated disability of mental disorders in four provinces in China during 2001-05: an epidemiological survey. Lancet 2009; 373: 2041-53.

4 World Health Organization. Mental Health Action Plan 2013-2020. WHO, 2013.

5 Charlson FJ, Baxter AJ, Cheng HG, Shidhaye R, whiteford HA. The burden of mental, neurological, and substance use disorders in China and India: a systematic analysis of community representative epidemiological studies. Lancet 2016; 388: 376-89.

6 Patel V, Xiao S, Chen H, Hanna F, Jotheeswaran AT, Luo D, et al. The magnitude of and health system responses to the mental health treatment gap in adults in India and China. Lancet 2016; 388: 3074-84.

7 Ran MS, Weng X, Chan CLW, Chen EY-H, Tang C-P, Lin F-R, et al. Different outcomes of never-treated and treated patients with schizophrenia: 14-year follow-up study in rural China. Br J Psychiatry 2015; 207: 495-500.

8 Prince M, Patel V, Shekhar S, Maj M, Maselko J, Phillips MR, et al. Global menta health 1 - No health without mental health. Lancet 2007; 370: 859-77.

9 Wang PS, Berglund P, Olfson M, Pincus HA, Wells KB, Kessler RC. Failure and delay in initial treatment contact after first onset of mental disorders in the National Comorbidity Survey Replication. Arch Gen Psychiatry 2005; 62: 603-13.

10 Saxena S, Thornicroft G, Knapp M, Whiteford H. Resources for mental health: scarcity, inequity, and inefficiency. Lancet 2007; 370: 878-89.

11 Gong P, Liang S, Carlton EJ, Jiang Q, Wu J, Wang L, et al. Urbanisation and health in China. Lancet 2012; 379: 843-52.

12 Xiang YT, Yu X, Sartorius N, Ungvari GS, Chiu HF. Mental health in China: challenges and progress. Lancet 2012; 380: 1715-6.

13 Clement S, Schauman O, Graham T, Maggioni F, Evans-Lacko S, Bezborodovs N et al. What is the impact of mental health-related stigma on help-seeking? A systematic review of quantitative and qualitative studies. Psychol Med 2015; 45: 11-27.

14 Andersen RM. Revisiting the behavioral model and access to medical care: does it matter? J Health Soc Behavior 1995; 36: 1-10.

15 Hudson CG. Socioeconomic status and mental illness: tests of the social causation and selection hypotheses. Am J Orthopsychiatry 2005; 75: 3-18.

16 Patel V, Araya R, Chatterjee S, Chisholm D, Cohen A, De Silva M, et al. Treatment and prevention of mental disorders in low-income and middle-income countries. Lancet 2007; 370: 991-1005.

17 Lund C, De Silva M, Plagerson S, Cooper S, Chisholm D, Das J, et al. Poverty and mental disorders: breaking the cycle in low-income and middle-income countries. Lancet 2011; 378: 1502-14.

18 Trani JF, Bakhshi P, Kuhlberg J, Narayanan SS, Venkataraman H, Mishra NN, et al. Mental illness, poverty and stigma in India: a case-control study. BMJ Open 2015; 5: e006355.

19 Das J, Do QT, Friedman J, Mckenzie D, Scott K. Mental health and poverty in developing countries: revisiting the relationship. Soc Sci Med 2007; 65: 467-80.

20 Ran MS, Chan CLW, Chen EYH, Mao W-J, Hu S-H, Tang C-P, et al. Differences in mortality and suicidal behaviour between treated and never-treated persons with schizophrenia in rural China. Br J Psychiatry 2009; 195: 126-31.

21 Ran MS, Chui CH, Wong IYL, Mao WJ, Lin FR, Liu B, et al. Family caregivers and outcome of people with schizophrenia in rural China: 14-year follow-up study. Soc Psychiatry Psychiatr Epidemiol 2016; 51: 513-20.

22 Xinjin Bureau of Statistics. Statistic Report of Xinjin Economic and Social Development 2015. Xinjin Bureau of Statistics, 2015.

23 Shen Y, Wang C. A Handbook of Epidemiological Investigation of Mental Illness. Renming Health Press, 1985.
24 World Health Organization. The ICD-10 Classification of Mental and Behavioural Disorders: Clinical Descriptions and Diagnostic Guidelines. WHO, 1992.

25 Wing JK, Cooper JE, Sartorius N. The Measurement and Classification of Psychiatric Symptoms. Cambridge University Press, 1974.

26 Ran MS, Xiang MZ, Huang MS, Shan Y. Natural course of schizophrenia: twoyear follow-up study in a rural Chinese community. Br J Psychiatry 2001; 178: $154-8$.

27 Ran MS, Xiang MZ, Chan CLW, Leff J, Simpson P, Huang MS, et al. Effectiveness of psychoeducational intervention for rural Chinese families experiencing schizophrenia: A randomised controlled trial. Soc Psychiatry Psychiatr Epidemiol 2003; 38: 69-75.

28 Janca A, Drimmelen J, Dittmann V, Isaac M, Ustun TB, et al. ICD-10 Symptom Checklist for Mental Disorders. WHO, 1994.

29 Ran MS, Mao WJ, Chan C, Chen EY, Conwell Y. Gender differences in outcomes in people with schizophrenia in rural China: 14-year follow-up study. $\mathrm{Br} \mathrm{J}$ Psychiatry 2015; 206: 283-8.

30 Ran MS, Xiao Y, Chui CHK, Hu XZ, Yu YH, Peng MM, et al. Duration of untreated psychosis (DUP) and outcome of people with schizophrenia in rural China: 14year follow-up study. Psychiatry Res 2018; 267: 340-5.

31 Ran MS, Zhang TM, Wong IYL, Yang X, Liu CC, Liu B, et al. Internalized stigma in people with severe mental illness in Rural China. Int J Soc Psychiatry 2018; 64: 9-16.

32 Ostrow L, Jessell L, Hurd M, Darrow SM, Cohen D. Discontinuing psychiatric medications: a survey of long-term users. Psychiatr Serv 2017; 68: 1232-8.

33 Papageorgiou G, Cañas F, Zink M, Rossi A. Country differences in patient characteristics and treatment in schizophrenia: data from a physician-based survey in Europe. Euro Psychiatry 2011; 26: 17-28.

34 Ran MS, Xiang MZ, Simpson P, Chan CLW. Family-Based Mental Health Care in Rural China. Hong Kong University Press, 2005.

35 Maura J, de Mamani AW. Mental health disparities, treatment engagement, and attrition among racial/ethnic minorities with severe mental illness: a review. J Clin Psychology in Med Settings 2017; 24: 187-210.

36 Nortje G, Oladeji B, Gureje $O$, Seedat S. Effectiveness of traditional healers in treating mental disorders: a systematic review. Lancet Psychiatry 2016; 3: 154-70.

37 Saraceno B, Levav I, Kohn R. The public mental health significance of research on socio-economic factors in schizophrenia and major depression. World Psychiatry 2005; 4: 181-5.

38 Ran $\mathrm{M}$, Lin FR, Huang D, Chan CLW, Li J, Mao WJ, et al. The family economic status and outcome of people with schizophrenia in Xinjin, Chengdu, China: 14-year follow-up study. Int J Soc Psychiatry 2017; 63: 203-11.

39 Kessler RC, Demler O, Frank RG, Olfson M, Pincus $H A$, Walters $E E$, et al. Prevalence and treatment of mental disorders, 1990 to 2003. New Eng J Med 2005; 352: 2515-23.

40 Demyttenaere K, Bruffaerts R, Posada-Villa J, Gasquet I, Kovess V, Lepine JP, et al. Prevalence, severity, and unmet need for treatment of mental disorders in the World Health Organization World Mental Health Surveys. JAMA 2004; 291: 2581-90.

41 Garrido MM, Kane RL, Kaas M, Kane RA. Perceived need for mental health care among community-dwelling older adults. J Gerontology 2009; 64(6): 704-12.

42 Ran MS, Xiang MZ, Conwell Y, Lamberti JS, Huang M-S, Shan $\mathrm{Y}-\mathrm{H}$, et al. Comparison of characteristics between geriatric and younger subjects with schizophrenia in community. J Psychiatr Res 2004; 38: 417-24.

43 Brenes GA, Danhauer SC, Lyles MF, Hogan PE, Miller ME. Barriers to mental health treatment in rural older adults. Am J Geriatr Psychiatry 2015; 23: 1172-8.

44 Andrade LH, Alonso J, Mneimneh Z, Wells JE, Al-Hamzawi A, Borges G, et al. Barriers to mental health treatment: results from the WHO World Mental Health surveys. Psychol Med 2014; 44: 1303-17.

45 Schomerus G, van der Auwera $\mathrm{S}$, Matschinger $\mathrm{H}$, Baumeister SE, Angermeyer MC. Do attitudes towards persons with mental illness worsen during the course of life? An age-period-cohort analysis. Acta Psychiatr Scand 2015; 132: 357-64.

46 Corrigan PW, Druss BG, Perlick DA. The impact of mental illness stigma on seeking and participating in mental health care. Psychol Sci in Pub Interest 2014; 15: $37-70$.

47 Dixon A, McDaid D, Knapp M, Curran C. Financing mental health services in low and middle income countries: equity and efficiency concerns. Health Policy Plan 2006; 21: 71-82.

48 Koenders JF, de Mooij LD, Dekker JM, Kikkert M. Social inclusion and relationship satisfaction of patients with a servere mental illness. Int J Soc Psychiatry 2017; 63: 773-81. 